\title{
Dexmedetomidine, an $\alpha$-2a adrenergic agonist, promotes ischemic tolerance in a murine model of spinal cord ischemia-reperfusion
}

\author{
Marshall T. Bell, MD, ${ }^{\text {, }}$ Ferenc Puskas, MD, PhD, ${ }^{b}$ Daine T. Bennett, MD, ${ }^{a}$ Paco S. Herson, PhD, \\ Nidia Quillinan, PhD, ${ }^{\mathrm{b}}$ David A. Fullerton, MD, ${ }^{\mathrm{a}}$ and T. Brett Reece, $\mathrm{MD}^{\mathrm{a}}$
}

\begin{abstract}
Objective: Dexmedetomidine, an $\alpha$-2a adrenergic agonist, given pre- and postoperatively was previously shown to attenuate neuronal injury in a murine model of spinal cord ischemia-reperfusion. In the brain, $\alpha-2$ agonists have been shown to induce the phosphorylation of cyclic AMP response-element binding protein (CREB), a transcription factor necessary for neuron survival. We hypothesized that the $\alpha$-2a adrenergic agonist given preoperatively increases CREB-mediated neuroprotective proteins, attenuating neuronal injury and cytoarchitectural decay.
\end{abstract}

\begin{abstract}
Methods: Mice (ie, C57BL/6 mice) underwent 5 minutes of aortic occlusion via median sternotomy. Mice received $25 \mu \mathrm{g} / \mathrm{kg}$ dexmedetomidine or equivalent normal saline at 24 hours, 12 hours, and 30 minutes preoperatively. Functional outcomes were recorded at 6 to 48 hours postoperatively when spinal cords were removed for histologic analysis. Spinal cords were examined for protein kinase B, CREB, B-cell lymphoma 2 , and brain-derived neurotrophic factor following treatment alone or ischemia-reperfusion surgery.
\end{abstract}

Results: Following aortic occlusion, mice in the treatment group had preserved neurologic function at all time points $(P<.05)$. Histologic analysis showed preserved cytoarchitecture and decreased neuronal injury in the treatment group when compared with ischemic controls. Additionally, analysis of spinal cord homogenate following surgery and pretreatment revealed a significant $(P<.05)$ increase in B-cell lymphoma 2 and brainderived neurotrophic factor expression and protein kinase B and CREB phosphorylation with $\alpha-2$ a adrenergic agonist pretreatment.

Conclusions: Pretreatment with the $\alpha$-2a agonist dexmedetomidine preserved neurologic function and attenuated neuronal injury following thoracic aortic occlusion in mice. This relationship was associated with an increased phosphorylation of protein kinase B and CREB and subsequent up-regulation of antiapoptotic factor B-cell lymphoma 2 and brain-derived neurotrophic factor. Thus, $\alpha$-2a receptor agonism-induced CREB phosphorylation and contributes to dexmedetomidine's protective mechanism in the spinal cord following ischemia. (J Thorac Cardiovasc Surg 2014;147:500-7)

The ischemic insult to the spinal cord during thoracoabdominal aortic interventions continues to have devastating consequences in up to one fifth of high-risk patients. ${ }^{1}$ Surgical adjuncts such as hypothermic circulatory arrest and cerebrospinal fluid drainage have reduced the incidence of the complication; however, implementation of these measures has only been met with a partial reduction of postoperative paraplegia. $^{2}$

Ischemia-reperfusion (IR) injury is complex and poorly understood. Neurons are the most vulnerable cells to

From the Department of Surgery ${ }^{\mathrm{a}}$ and Department of Anesthesiology, ${ }^{\mathrm{b}}$ School of Medicine, University of Colorado at Denver, Aurora, Colo.

Disclosures: Authors have nothing to disclose with regard to commercial support.

Read at the 93rd Annual Meeting of The American Association for Thoracic Surgery, Minneapolis, Minnesota, May 4-8, 2013.

Received for publication May 2, 2013; revisions received July 14, 2013; accepted for publication July 18, 2013; available ahead of print Sept 16, 2013.

Address for reprints: Marshall T. Bell, MD, Department of Surgery, University of Colorado School of Medicine, 12631 E 17th Ave, Mail Stop C310, Room 6601,

Aurora, CO 80045 (E-mail: Marshall.bell@ucdenver.edu).

$0022-5223 / \$ 36.00$

Copyright $\Subset 2014$ Published by Elsevier Inc. on behalf of The American Association for Thoracic Surgery

http://dx.doi.org/10.1016/j.jtcvs.2013.07.043 ischemia and coupled with their inability to regenerate, spinal cord IR is a significant threat to patients. Pharmacologic adjuncts that increase ischemic tolerance by neurons would serve as an ideal adjunct to further reduce the risk of this complication.

The neuroprotective effects of $\alpha-2$ receptor agonists have been studied for more than 20 years $^{3}$; however, their mechanisms of protection from ischemia-reperfusion remain unknown. In cerebral neurons, clonidine and other $\alpha-2$ agonists have been shown to promote phosphorylation of neuroprotective pathways. ${ }^{4}$ One such pathway converges at cyclic AMP response-element binding protein (CREB). ${ }^{5,6}$ CREB is a transcription factor that is critical for neuron survival after ischemia supporting its role in the induction of ischemic tolerance. ${ }^{7}$ Among other functions, CREB induces expression of prosurvival proteins such as B-cell lymphoma 2 (BCL-2), ${ }^{8}$ brain derived neurotrophic factor (BDNF), ${ }^{9,10}$ and a variety of free radical scavengers. ${ }^{11,12}$

Dexmedetomidine is a newer and more specific $\alpha-2 \mathrm{a}$ agonist. It is commonly used in operating rooms and in 


\author{
Abbreviations and Acronyms \\ BCL-2 = B-cell lymphoma 2 \\ $\mathrm{BDNF}=$ brain-derived neurotrophic factor \\ $\mathrm{CREB}=$ cyclic AMP response-element binding \\ protein \\ IR = ischemia-reperfusion \\ PBS $=$ phosphate buffered saline
}

intensive care units. It has gained widespread attention for its neuroprotective properties in the brain. ${ }^{13,14} \mathrm{We}$ therefore postulated that it may have neuroprotective properties in the spinal cord. We hypothesized that pretreatment with dexmedetomidine will preserve neurologic function and neuron viability following thoracic aortic occlusion by promoting ischemic tolerance in the spinal cord through an induction of CREBmediated neuroprotective proteins.

\section{METHODS}

\section{Animal Procedures}

The Animal Care and Use Committee at the University of Colorado at Denver Health Sciences Center approved all experiments, and this investigation conformed to the Guide for the Care and Use of Laboratory Animals published by the National Institutes of Health (www.nap.edu/catalog/5140. html). Male C57BL/6 mice between ages 10 and 20 weeks were used for all experiments.

\section{Aortic Crossclamping: IR Surgery}

Mice were anesthetized using $2 \%$ isoflurane and placed in the supine position. Surgery was performed under normothermic conditions. The core body temperature was maintained at $36.5^{\circ} \mathrm{C} \pm 0.5^{\circ} \mathrm{C}$ using a rectal temperature probe and automatic temperature adjusting bed (Vestavia Scientific, Birmingham, Ala). The aortic arch was exposed using a cervicothoracic approach as previously described. ${ }^{15}$ Disruption of arterial flow to the spinal cord was achieved by placing vascular clamps on the aortic arch distal to the left common carotid artery and the subclavian artery for 5 minutes. To confirm at least $90 \%$ reduction in distal flow, a laser Doppler blood flow monitor (Moor Instruments, Devon, United Kingdom) was placed over the left femoral artery.

\section{Medication Administration}

Mice in the treatment group received intraperitoneal injections of $25 \mu \mathrm{g} / \mathrm{kg}$ dexmedetomidine $(\mathrm{n}=8)$ based on previous studies ${ }^{13}$ or normal saline $(\mathrm{n}=8)$ at 24 hours, 12 hours, and 30 minutes before IR. Additionally, 3 mice underwent sham surgery with sternotomy and dissection of the aorta and subclavian artery with no occlusion. To compare effects of medication administration alone, 4 treated mice and 4 mice given normal saline injections at time points above had spinal cords removed for protein and messenger RNA studies without IR surgery.

\section{Functional Scores}

The Basso Mouse Scale for locomotion, ${ }^{16}$ which ranges from a score of 0 for complete paraplegia to a score of 9 for normal function, was used to quantify hind-limb function in mice after ischemia. Function was scored at $12,24,36$, and 48 hours after reperfusion.

\section{Histologic Analysis}

After 48 hours of reperfusion, the animals were sacrificed. The vertebral column was removed en bloc from T8-L3. Spinal cords were removed from the vertebral column by injection of phosphate buffered saline (PBS) $(\mathrm{pH}$ 7.4) into the spinal column. Spinal cords were then transferred to $4 \%$ formalin where they remained for at least 24 hours before paraffin embedding, sectioning, and hemotoxylin and eosin staining.

\section{Quantification of Neuron Degeneration}

Spinal cords were placed into paraffin block following 48 hours of reperfusion. Sections 5- $\mu \mathrm{m}$ thick were placed on poly-lysine slides. Paraffin was removed by placing slides in $100 \%$ xylene followed by washes with $100 \%$ ethanol and $70 \%$ ethanol deionized water. Slides were then blocked with a solution of $0.06 \%$ potassium permanganate (Sigma Aldrich, St Louis, Mo) and stained with Flouro Jade-B stain (EDM Millipore, Billerica, Mass), a marker for neuron degeneration. ${ }^{17}$ Stock solution was diluted in $0.1 \%$ acetic acid a final concentration of $0.0004 \%$ Flouro Jade-B and applied for 30 minutes. Slides were then rinsed in deionized water and placed in the dark to air-dry overnight. Once dry, $100 \%$ xylene was applied and slides were mounted with DPX (Sigma Aldrich, St Louis, Mo). Quantification of degenerating neurons was performed by a blinded observer and the number and expressed as number of degenerating neurons per high power field.

\section{Immunoblotting}

Spinal cords were removed following pretreatment or IR surgery (Figure 1). Protein extracts were obtained using M-lysis buffer (Roche, Indianapolis, Ind) per the manufacturer's instructions and were placed in a $4 \mathrm{X}$ Laemmmli buffer with $\beta$-mercaptoethanol and boiled for 10 minutes at $100^{\circ} \mathrm{C}$, loaded into a 15 -well $4 \%$ to $20 \%$ gradient-ready gels (Bio-Rad, Hercules, Calif), and run at $160 \mathrm{~V}$ for 450 minutes. The gels were transferred to nitrocellulose membranes at $100 \mathrm{~V}$ for 60 minutes and then cross-linked using a UV Stratalinker (Stratagene, La Jolla, Calif). The membranes were blocked in $5 \%$ dry milk in $0.1 \%$ Tween with PBS, and rinsed 3 times in $0.1 \%$ Tween with PBS. The blocked membranes were incubated with primary antibodies overnight at $4{ }^{\circ} \mathrm{C}(5 \%$ bovine serum albumin in $0.1 \%$ Tween with PBS). The membranes were washed in $0.1 \%$ Tween with PBS 3 times and incubated in appropriate horseradish peroxidase-conjugated secondary antibodies diluted to $1: 5000$ in $5 \%$

\begin{tabular}{l} 
Spinal Cord Removal at 24 hours \\
AKT, CREB, BCL2, BDNF \\
\hline Dex Pretreatment
\end{tabular}

FIGURE 1. Graphic representation of the study design. AKT, Protein kinase B; $C R E B$, cyclic AMP response-element binding protein; $B C L 2$, B-cell lymphoma 2; $B D N F$, brain-derived neurotrophic factor; Dex, dexmedetomidine. 


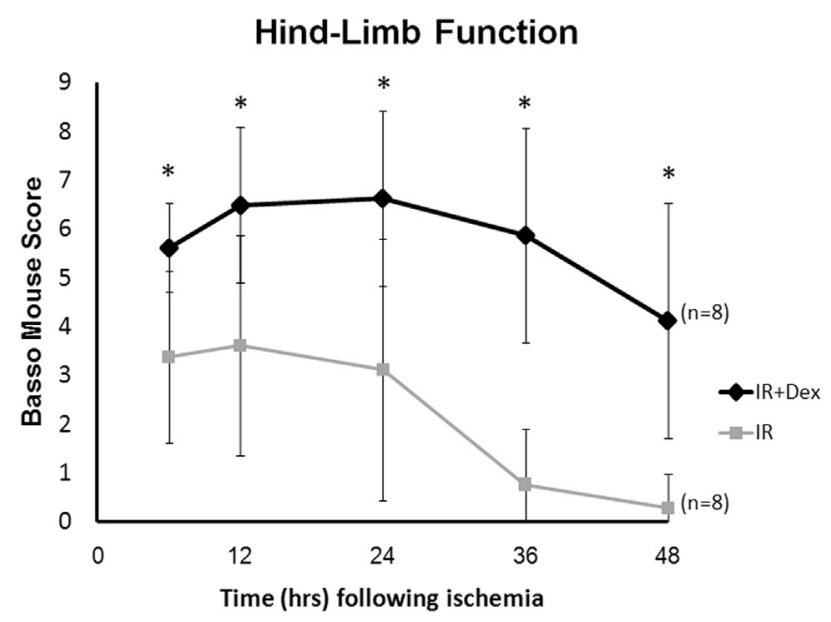

FIGURE 2. Following ischemia-reperfusion (IR) surgery with 5 minutes of aortic occlusion a significant functional deficit in hind-limb function was observed in all mice. Twenty-four hours of pretreatment with dexmedetomidine $(I R+D e x)$ resulted in significantly higher neurologic function at all time points postoperatively. Error bars represent standard error of the mean. $* P<.05$.

bovine serum albumin in $0.1 \%$ Tween with PBS for 1 hour at room temperature. SuperSignal West Dura Chemiluminescent Substrate for HRP (Thermo Scientific, Rockford, Ill) was used for 5 minutes; membranes were visualized using a Chemidoc Hi Sensitivity scanner (BioRad, Hercules, Calif). Mean density values of bands were quantified by QuantityOne software (BioRad, Hercules, Calif).

\section{Quantitative Polymerase Chain Reaction}

Measurements of BCL-2 and BDNF transcripts from treated and untreated spinal tissue without undergoing IR surgery were performed. Total RNA was isolated using the RNAqueous-4 PCR kit (Ambion, Austin, Tex) per the manufacturer's instructions. Real-time polymerase chain reactions using iQ mastermix (Bio-Rad, Hercules, Calif) on Bio-Rad CFX detection system (Hercules, Calif) were performed in duplicate using $50 \mathrm{ng}$ complementary DNA. FAM-labeled probe/primer sets used to detect BCL-2 (Mm00477631_m1) and BDNF (Mm04230607_s1) were synthesized by Invitrogen (Grand Island, NY). The housekeeping gene $\beta$-actin (Mm00607939_s1) was also assayed for each sample using $5 \mathrm{ng}$ complementary DNA. Cycle parameters used $95^{\circ} \mathrm{C}$ for 3 minutes followed by 40 cycles of $95^{\circ} \mathrm{C}$ for 15 seconds and $60^{\circ} \mathrm{C}$ for 1 minute. Expression levels were calculated using the delta-delta $\mathrm{Ct}$ method relative to $\beta$-actin.

\section{Statistical Analysis}

Statistical analysis was performed using repeated measures and the Kruskal-Wallis nonparametric analysis of variance with Bonferroni/Dunn post-hoc test using StatView (SAS Institute Inc, Cary, NC). Two-way analysis was performed using unpaired $t$ test. Continuous data are presented as mean \pm standard error. Continuous data are represented as mean \pm standard deviation.

\section{RESULTS \\ Functional Outcomes}

Mice in the sham group had no observable functional deficit at any time point in the postoperative period. Ischemia with 5 minutes of aortic occlusion produced a significant functional deficit in all mice. Twenty-four hours of pretreatment with dexmedetomidine resulted in a significant preservation of neurologic function $(P<.05)$ at all time points postoperatively (Figure 2).

\section{Histologic and Neurocytoarchitectural Outcomes}

All mice undergoing IR surgery had a disruption of anterior horn cytoarchitecture when compared with sham mice. Ischemic control mice demonstrated a more pronounced disruption of cytoarchitcture, a reduction of normal-appearing anterior horn neurons and increased vacuolization when compared with the sham and treatment groups (Figure 3). Anterior horns of spinal cords were stained with Flouro Jade-B, a marker of neuronal injury, and quantified by a blinded observer (Figure 4). All mice undergoing IR surgery had a significant increase in Flouro Jade-B-positive neurons. Mice treated with dexmedetomidine had a significant reduction in Flouro Jade-B-positive neurons when compared with ischemic controls.

\section{Pretreatment and Survival Signaling}

To examine spinal cord cellular signaling at time point 0 , mice received identical pretreatment with dexmedetomidine $(\mathrm{n}=4)$ or $0.9 \%$ normal saline $(\mathrm{n}=4)$ and had spinal cords removed with no surgery. Spinal cord homogenates were analyzed for AKT and CREB phosphorylation. Following pretreatment there was a robust and significant $(P<.05)$ increase in AKT and CREB phosphorylation

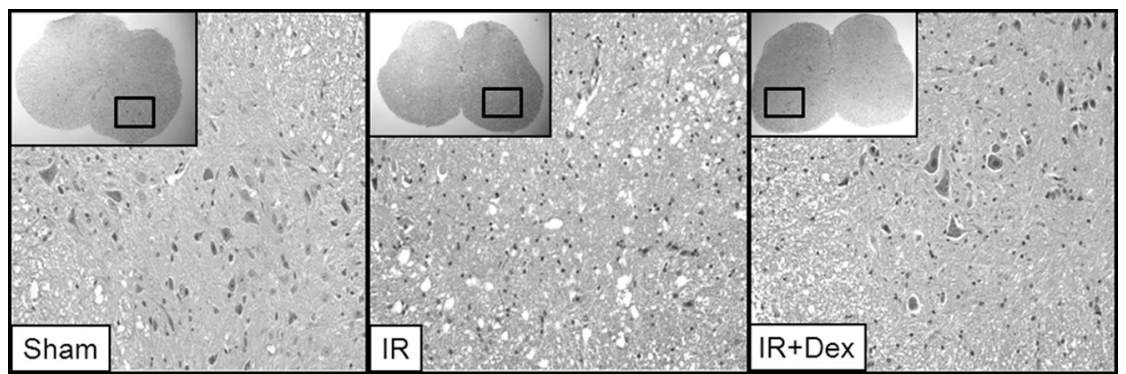

FIGURE 3. Mice undergoing ischemia-reperfusion surgery had a significant disruption of cytoarchitecture when compared with sham mice. A reduction of normal-appearing anterior horn neurons and increased vacuolization was observed in ischemic control mice (IR) when compared with the sham and treatment $(I R+D E X)$ groups. 


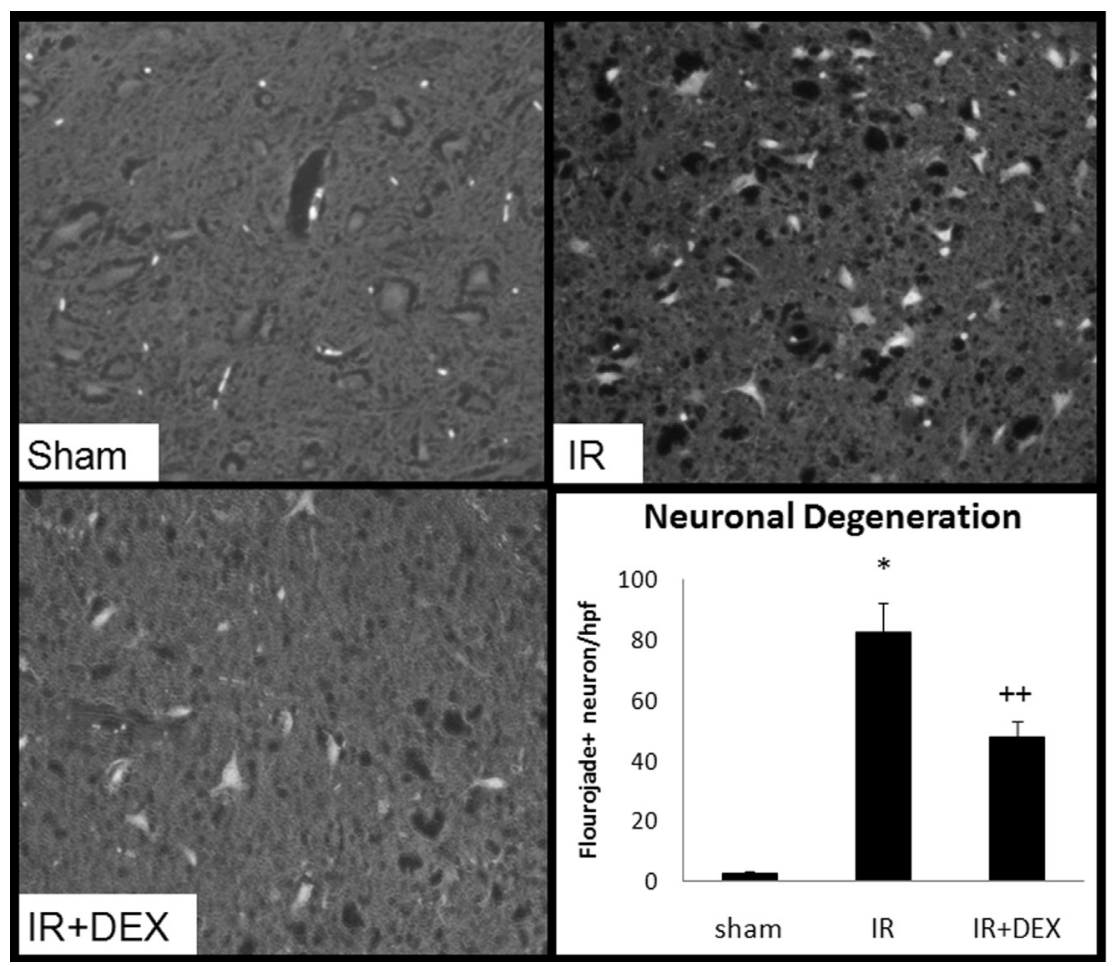

FIGURE 4. All mice undergoing ischemia-reperfusion surgery had a significant increase in Flouro Jade-B (EDM Millipore, Billerica, Mass)-positive neurons. Mice treated with dexmedetomidine $(I R+D E X)$ had a significant reduction in neuronal injury when compared with ischemic controls $(I R)$. Error bars represent standard error of the mean. $* P<.001 .++P<.0001$.

with treatment alone (Figure 5). To determine if CREB phosphorylation resulted in a subsequent production of neuroprotective proteins with dexmedetomidine treatment alone, spinal cord tissue was tested by quantitative polymerase chain reaction for production of BCL-2 and BDNF. Pretreatment with dexmedetomidine resulted in a significant up-regulation of BCL-2 and BNDF at time point 0 when compared with vehicle controls (treated with $0.9 \%$ normal saline) (Figure $6, A$ ). At the duration of the experiment spinal cord homogenate was examined for the presence of CREB-mediated proteins BCL-2 and BDNF. Dexmedetomidine treatment resulted in statistically significant elevations in both BCL-2 and BDNF expression following IR surgery (Figure 6, $B$ and $C$ ).

\section{DISCUSSION}

Paraplegia remains the most-feared complication of aortic surgery. The results of our study demonstrate that treatment with the $\alpha$-2a agonist dexmedetomidine before aortic occlusion provided functional protection. This functional protection was associated with histologic evidence of preservation of the spinal cord cytoarchitecture, diminished marker of neuronal injury (Flouro Jade-B), activation of neuron signaling pathways that are important for neuron survival (protein kinase $B$ and CREB signaling), and increased production of CREB-mediated proteins linked to neuron survival (BCL-2 and BDNF).
These data suggest that pretreatment with dexmedetomidine may offer a strategy for spinal cord protection during aortic surgery.

Using a murine model of delayed paraplegia, our laboratory has previously shown that dexmedetomidine can produce significant neuroprotection in the spinal cord when given before and after thoracic aortic occlusion. ${ }^{18}$ The murine model used in our study was designed to mimic the early paraplegia seen following aortic surgery. It is widely held that such early paraplegia results from a severe metabolic injury to the spinal cord neurons during ischemia. ${ }^{19}$ Therefore, the model used in our study differs from that which our group has previously used in 2 important ways. First, a longer ischemic time was employed. In our model this produced an injury that more closely resembled the clinical entity of immediate paraplegia. ${ }^{12}$ Second, no postoperative treatment was included to focus on the potential actions of pretreatment with an $\alpha$-2a agonist.

The cumulative injuries of ischemia and reperfusion are complex, and those of reperfusion are known to exacerbate those of ischemia. In our study, the $\alpha-2 \mathrm{a}$ agonist was administered before ischemia. A limitation of our study is that it is not possible to determine if the beneficial effects of dexmedetomidine were due to attenuation of the ischemic injury, the reperfusion injury, or both. Nonetheless, when given before ischemia, dexmedetomidine exhibited functional protection against IR. 

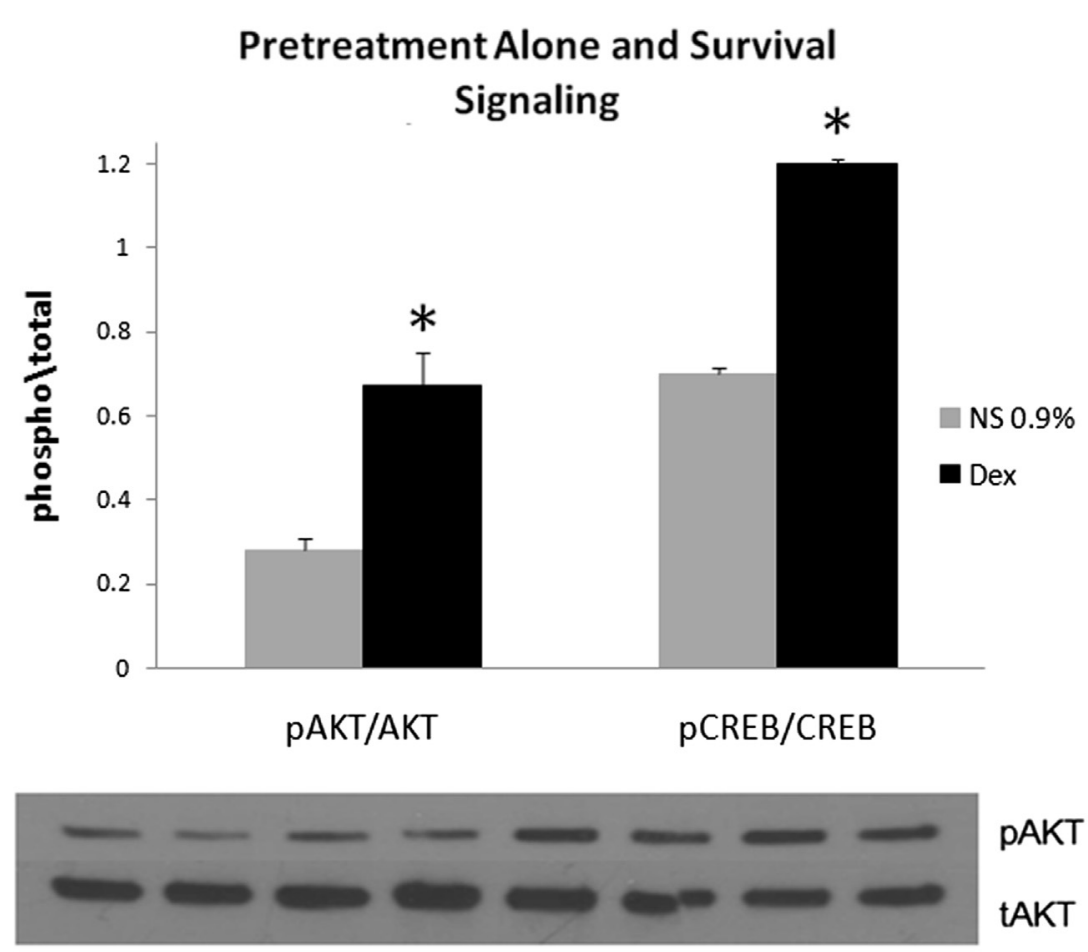

\section{pAKT \\ tAKT}

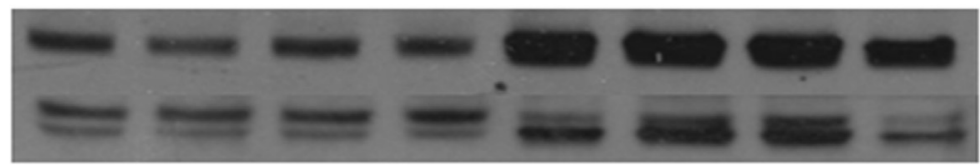

pCREB

tCREB

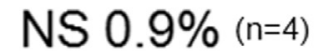

$\operatorname{Dex}(n=4)$

FIGURE 5. Spinal cord cellular signaling of neuroprotective pathways at time point 0 . Mice receiving pretreatment alone with dexmedetomidine (Dex) had significantly higher phosphorylation of prosurvival protein kinase $(p A K T)(P=.005)$ and prosurvival cyclic AMP response-element binding protein $(p C R E B)(P=.004)$ in all patients $P$ values of $<0.05$ are denoted by “*” when compared with mice receiving normal saline $(N S) 0.9 \%$. $* P<$ .05. Error bars represent standard error of the mean. $t A K T$, Total protein kinase B; $t C R E B$, total cyclic amp response element binding protein.

The period of ischemia in our study produced a significant spinal cord injury immediately following surgery and throughout the duration of the experiment in control mice. In comparison, mice in the dexmedetomidine-treatment group had significantly preserved hind-limb function when compared with ischemic control at all time points through the duration of the experiment. Though the extent of neuroprotection was not as complete as we have seen with shorter durations of ischemic times ${ }^{18}$; however, statistical significance was still obtained. These findings could be secondary to either the increased ischemic time or absence of postoperative dexmedetomidine administration.

Our second purpose was to determine if dexmedetomidine pretreatment resulted in activation neuronal prosurvival pathways. This was accomplished by examining spinal cords at time point 0 for induction of neuroprotective pathways related to the promotion of ischemic tolerance, namely protein kinase $\mathrm{B}^{20}$ and CREB. ${ }^{7}$ Dexmedetomidine treatment resulted in a significant increase in phosphorylation of both protein kinase B and protein kinase B's downstream target CREB.

CREB is a transcription factor considered to be critical in programmed cell survival, as opposed to death. ${ }^{21}$ Additionally, it is widely accepted that CREB phosphorylation is a necessary component of ischemic preconditioning. ${ }^{22}$ In stroke models, CREB-mediated induction of protective pathways significantly preserved neuronal survival. ${ }^{6}$ More importantly, studies on global ischemia have intimated that robust increases in CREB phosphorylation in neurons have a substantial affect on ischemic tolerance when compared with ischemia-vulnerable neurons. ${ }^{23,24}$

The protective effects of CREB likely results from the induction of neuroprotective proteins. Both BCL-2 and BDNF have been shown to be induced by CREB. ${ }^{8,9}$ BCL2 is a potent inhibitor of neuron death from apoptosis and necrosis. ${ }^{25}$ Following ischemia, BCL-2 prevents mitochondrial membrane permeability, inhibiting cytochrome $\mathrm{C}$ release. ${ }^{26}$ Studies of transient brain ischemia demonstrated 


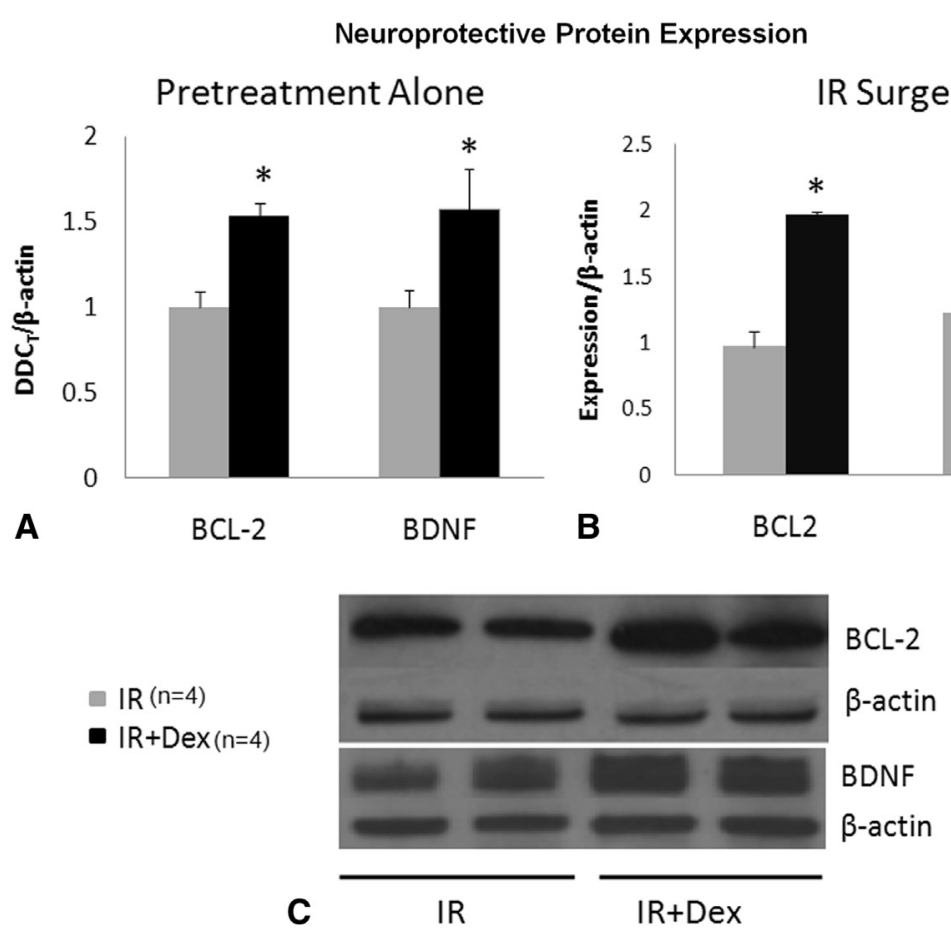

FIGURE 6. A, Quantitative polymerase chain reaction for production of B-cell lymphoma $2(B C L-2)$ and brain-derived neurotrophic factor $(B D N F)$ transcripts was performed. Pretreatment with dexmedetomidine resulted in a significant up-regulation of BCL-2 $(P \leq .0001)$ and BNDF $(P=.040)$ messenger RNA expression at time point 0 . B, Expression of cyclic AMP response-element binding-mediated neuroprotective proteins BCL-2 and BDNF was examined 48 hours following ischemia-reperfusion $(I R)$ surgery and compared with housekeeping protein $\beta$-actin. Significant elevations in both BCL-2 $(P=.004)$ and BDNF $(P=.0035)$ expression following IR surgery were observed with dexmedetomidine pretreatment $(I R+D e x)$ when compared with ischemic controls (IR) 48 hours following surgery. C, Representative immunoblot following IR. Error bars represent standard error of the mean. $D D C_{T}$, Expression.

that overexpression of BCL-2 significantly improved stroke outcomes. ${ }^{27}$ Moreover, similar overexpression of BCL-2 in the spinal cord suppressed motor functional deficits following traumatic injury. ${ }^{28}$

Another protein, BDNF, is a member of the neurotrophin family. Neurotrophins are a family of proteins that can regulate the survival, development, and function of neurons. ${ }^{21}$ BDNF may have a role in neuroplasticity related to improved long-term outcomes in stroke models. It has also been associated with inflammatory reduction and reduction of apoptosis. ${ }^{29}$ No matter the mechanism, BDNF overexpression has been shown to induce a critical reduction of neuronal injury following cerebral ischemia. ${ }^{30}$ Pretreatment with dexmedetomidine resulted in an increased expression of messenger RNA from the CREBmediated proteins BCL-2 and BDNF. Following IR, the levels of these proteins were also significantly elevated in the treated mice with preserved function providing further evidence of neuroprotection.

The results of our study suggest that dexmedetomidine may be a clinical accessible tool to be used in aortic surgery. Unlike other agents with reported neuroprotective properties, dexmedetomidine is commonly used clinically in both operating rooms and intensive care units. Additionally, dexmedetomidine does not influence evoked potential as some of the anesthetics can. ${ }^{31}$ Dexmedetomidine can readily be added as an adjunct during high-risk aortic interventions.

Our study is not without limitations. First, in animal models it is difficult to distinguish the injuries resulting from the ischemic and reperfusion insults. Second, although the study provides a mechanistic explanation for dexmedetomidine mechanism of action, additional studies using CREB inhibitors in different central nervous system cell lineages will provide further information for dexmedetomidine's neuroprotective effects.

\section{CONCLUSIONS}

Pretreatment with the $\alpha$-2a agonist dexmedetomidine preserves neurologic function and attenuated neuronal injury following thoracic aortic occlusion in mice. The observed neuroprotection was associated with CREB -phosphorylation and production of CREB's downstream neuroprotective targets BCL-2 and BDNF. Dexmedetomidine may be another adjunct for reducing the devastating complication of paraplegia in high-risk aortic interventions. 


\section{References}

1. Greenberg RK, Lu Q, Roselli EE, Svensson LG, Moon MC, Hernandez AV, et al. Contemporary analysis of descending thoracic and thoracoabdominal aneurysm repair: a comparison of endovascular and open techniques. Circulation. 2008; 118:808-17.

2. Okita Y. Fighting spinal cord complication during surgery for thoracoabdominal aortic disease. Gen Thorac Cardiovasc Surg. 2011:59:79-90.

3. Hoffman WE, Cheng MA, Thomas C, Baughman VL, Albrecht RF. Clonidine decreases plasma catecholamines and improves outcome from incomplete ischemia in the rat. Anesth Analg. 1991;73:460-4.

4. Zhu Y-M, Wang C-C, Chen L, Qian L-B, Ma L-L, Yu J, et al. Both PI3K/Akt and ERK1/2 pathways participate in the protection by dexmedetomidine against transient focal cerebral ischemia/reperfusion injury in rats. Brain Res. 2013;1494:1-8.

5. Patel NJ, Chen MJ, Russo-Neustadt A. Norepinephrine and nitric oxide promote cell survival signaling in hippocampal neurons. Eur J Pharmacol. 2010;633:1-9.

6. Kitagawa K. CREB and cAMP response element-mediated gene expression in the ischemic brain. FEBS J. 2007;274:3210-7.

7. Hara T, Hamada J, Yano S, Morioka M, Kai Y, Ushio Y. CREB is required for acquisition of ischemic tolerance in gerbil hippocampal CA1 region. J Neurochem. 2003;86:805-14

8. Ji L, Mochon E, Arcinas M, Boxer LM. CREB proteins function as positive regulators of the translocated BCL-2 allele in $\mathrm{t}(14 ; 18)$ lymphomas. J Biol Chem. 1996;271:22687-91.

9. Conti AC, Cryan JF, Dalvi A, Lucki I, Blendy JA. cAMP response elementbinding protein is essential for the upregulation of brain-derived neurotrophic factor transcription, but not the behavioral or endocrine responses to antidepressant drugs. J Neurosci. 2002;22:3262-8.

10. Finkbeiner S, Tavazoie SF, Maloratsky A, Jacobs KM, Harris KM, Greenberg ME. CREB: a major mediator of neuronal neurotrophin responses. Neuron. 1997;5:1031-47.

11. Liu N, Yu Z, Li Y, Yuan J, Zhang J, Xiang S, et al. Transcriptional regulation of mouse Neuroglobin gene by cyclic AMP responsive element binding protein (CREB) in N2a cells. Neurosci Lett. 2013;534:333-7.

12. Krönke G, Bochkov VN, Huber J, Gruber F, Blüml S, Fürnkranz A, et al. Oxidized phospholipids induce expression of human heme oxygenase-1 involving activation of cAMP-responsive element-binding protein. J Biol Chem. 2003;278:51006-14.

13. Ma D, Hossain M, Rajakumaraswamy N, Arshad M, Sanders RD, Franks NP, et al. Dexmedetomidine produces its neuroprotective effect via the alpha 2Aadrenoceptor subtype. Eur J Pharmacol. 2004;502:87-97.

14. Sato K, Kimura T, Nishikawa T, Tobe Y, Masaki Y. Neuroprotective effects of a combination of dexmedetomidine and hypothermia after incomplete cerebral ischemia in rats. Acta Anaesthesiol Scand. 2010;54:377-82.

15. Smith PD, Puskas F, Meng X, Cho D, Cleveland JC, Weyant MJ, et al. Ischemic dose-response in the spinal cord: both immediate and delayed paraplegia. J Surg Res. 2011;7:1-7.

16. Basso DM, Fisher LC, Anderson AJ, Jakeman LB, McTigue DM, Popovich PG. Basso Mouse Scale for locomotion detects differences in recovery after spinal cord injury in five common mouse strains. J Neurotrauma. 2006;23:635-59

17. Schmued LC, Hopkins KJ. Fluoro-Jade B: a high affinity fluorescent marker for the localization of neuronal degeneration. Brain Res. 2000;874:123-30

18. Bell MT, Puskas F, Smith PD, Agoston VA, Fullerton DA, Meng X, et al. Attenuation of spinal cord ischemia-reperfusion injury by specific $\alpha$-2a receptor activation with dexmedetomidine. J Vasc Surg. 2012;56:1398-402.

19. Won SJ, Kim DY, Gwag BJ. Cellular and molecular pathways of ischemic neuronal death. J Biochem Mol Biol. 2002;35:67-86.

20. Zhao H, Sapolsky RM, Steinberg GK. Phosphoinositide-3-kinase/akt survival signal pathways are implicated in neuronal survival after stroke. Molec Neurobiol. 2006;34:249-70

21. Finkbeiner S. Creb couples neurotrouphin signals to survival messages. Neuron. 2000;25:11-4.

22. Hu BR, Fux CM, Martone ME, Zivin JA, Ellisman MH. Persistent phosphorylation of cyclic AMP responsive element-binding protein and activating transcription factor-2 transcription factors following transient cerebral ischemia in rat brain. Neuroscience. 1999;89:437-52.

23. Lee H-T, Chang Y-C, Wang L-Y, Wang S-T, Huang C-C, Ho C- J. cAMP response element-binding protein activation in ligation preconditioning in neonatal brain. Ann Neurol. 2004;56:611-23.

24. Gidday JM. Cerebral preconditioning and ischaemic tolerance. Nat Rev Neurosci. 2006;7:437-48

25. Kane DJ, Ord T, Anton R, Bredesen DE. Expression of bcl-2 inhibits necrotic neural cell death. J Neruosci Res. 1995;40:269-75.
26. Soane L, Fiskum G. Inhibition of mitochondrial neural cell death pathways by protein transduction of Bcl-2 family proteins. J Bioenerg Biomembr. 2005;37: 179-90

27. Kitagawa K, Matsumoto M, Tsujimoto Y, Ohtsuki T, Kuwabara K, Matsushita K, et al. Amelioration of hippocampal neuronal damage after global ischemia by neuronal overexpression of BCL-2 in transgenic mice. Stroke. 1998;29:2616-21.

28. Wang Y, Sun Z, Zhang K, Xu G, Li G. Bcl-2 in suppressing neuronal apoptosis after spinal cord injury. World J Emerg Med. 2011;2:38-44

29. Chen A, Xiong LJ, Tong Y, Mao M. The neuroprotective roles of BDNF in hypoxic ischemic brain injury. Biomed Rep. 2012;167-76.

30. Shi Q, Zhang P, Zhang J, Chen X, Lu H, Tian Y, et al. Adenovirus-mediated brain-derived neurotrophic factor expression regulated by hypoxia response element protects brain from injury of transient middle cerebral artery occlusion in mice. Neurosci Lett. 2009;465:220-5

31. Bala E, Sessler DI, Nair DR, McLain R, Dalton JE, Farag E. Motor and somatosensory evoked potentials are well maintained in patients given dexmedetomidine during spine surgery. Anesthesiology. 2008;109:417-25.

\section{Discussion}

Dr John S. Ikonomidis (Charleston, SC). I would like to congratulate Dr Bell for a very nice presentation and thank him for sending me his slides and his presentation in advance. I have a few questions. The first relates to the model. Conceptually speaking in humans, 5 minutes of aortic occlusion really would not be expected to produce the kind of spinal cord damage that you saw in this model, so please explain briefly why you think there are differences between mice and humans and put that in the context of the potential clinical relevance of this study.

Dr Bell. I think 1 of the main differences within the spinal cords is that the murine metabolic rate is much higher, so we are able to see this injury under normothermic conditions at 5 minutes compared with 45 minutes.

Dr Ikonomidis. While we are on the subject of hemodynamics and heart rate, did you measure blood pressure during this study and can you comment on blood pressure between the 2 groups?

Dr Bell. We previously looked at blood pressure with dexmedetomidine treatment before and after surgery, and did not see a difference in mice that were under the influence of isoflurane anesthesia.

Dr Ikonomidis. What was the justification for the use of $25 \mathrm{mg}$ dexmedetomidine?

Dr Bell. It was done based on previous studies of neuroprotection with the middle cerebral artery occlusion model. It was seen that the neuroprotective effects peaked at $25 \mu \mathrm{g} / \mathrm{kg}$. This was also a dose that caused no sedation and, we think, more correlated with an anxiolytic dose.

Dr Ikonomidis. So if I were to give it to human patients, what would be the intravenous dose?

Dr Bell. That is a great question. We haven't been able to quantify the exact amount for human patients. The intravenous dose is 1 $\mathrm{mg} / \mathrm{kg}$ loaded over 10 minutes and then $0.5 \mathrm{mg} / \mathrm{kg}$ basal dosing.

Dr Ikonomidis. It strikes me that a lot of the data you presented here are, to a large extent, associative, and I was wondering if you have done specific mechanistic studies. For example, do you have a cyclic AMP response-element binding (CREB) protein knockout mouse in which you can abrogate spinal cord damage, or have you done similar studies?

Dr Bell. CREB knockout mice are nonviable, but we have developed our in vitro model, and we are able to grow these neurons in a dish. We will be able to then use antibodies as well as 
nucleotide inhibitors that we can further study to be able to nail down that mechanism. These studies are going on currently.

Dr Ikonomidis. It is interesting that this model causes both initial and delayed neurologic damage, so it mimics initial paraplegia but also delayed paraplegia, and they both seem to be inhibited by dexmedetomidine. Can you comment on the potential mechanisms? Are they similar or the same?

Dr Bell. In this experiment we chose 5 minutes because we wanted to look at the initial ischemic insult and be able to associate the observed injury with the ischemic insult. We, as well as others, have looked at microglial activation and their contributions to delayed paralysis. Dexmedetomidine also has some anti-inflammatory effects on microglial in vitro that could also contribute to these findings. We did not treat them postoperatively because we did not want to modify the inflammatory cascade in any way.

Dr Ikonomidis. You measured protein kinase B (AKT) levels in this study; an abundance of AKT. Did you look at any of its activating surrogates, such as phosphoinositide 3-kinase?

Dr Bell. No, we have not looked at that. It is something we want to do in vitro and to tie in more closely to the $\alpha$-2 receptor, and I think in vitro we could more easily do that.

Dr Ikonomidis. Thank you very much.

Dr Scott A. LeMaire (Houston, Tex). Dr Ikonomidis touched on this, but my question relates to the potential use for this agonist for not only treating acute injury we see early in the operating room but also delayed injury. How long do the cellular effects of this agonist persist? Do you have any data related to how long the protective effects might last?

Dr Bell. We want to carry it out further in our murine model and look at some of the chronic changes. We have observed that delayed paraplegia consistently occurs at 36 to 48 hours. We have seen protection against delayed paraplegia as well when we post-treated mice at reperfusion in a previous study. One of the potential benefits of this drug is that you can keep a high-risk patient, maybe an Extent 2 patient, in an intensive care unit on this drug even when they are extubated on an anxiolytic dosing regimen.

Dr Pirooz Eghtesady (St Louis, Mo). I have a question related to the 24-hour pretreatment. How did you come up with 24 hours as opposed to 12 hours or a week, and how would you translate that to the clinical setting?

Dr Bell. We chose the pretreatment interval based on previous models of ischemic preconditioning that showed a maximal effect when the initial surgery of the sublethal ischemia was performed at 24 hours. We also believed that this interval would give us an adequate time period for the expression of neuroprotective proteins. In the clinical setting I think it would not be out of the question to admit a patient the day before for an infusion of dexmedetomidine at an anxiolytic dose. 